\title{
TEMPERATURE NON-HOMOGENEIETIES IN A CATALYTIC REACTOR WITH A PERIODIC CHANGE IN THE DIRECTION OF THE REACTION MIXTURE FEED
}

\author{
IVANKA ZHELEVA \\ Ruse University, \\ 8 Studentska str., 7017 Ruse, Bulgaria, \\ e-mail: izheleva@uni-ruse.bg
}

[Received 05 February 2015. Accepted 15 June 2015]

\begin{abstract}
Temperature non-homogeneities in a catalytic reactor with periodic change in the direction of the reaction mixture feed is investigated in the present work. The temperature of the reaction mixture is described using a numerical algorithm for simulation of the work of the catalytic reactor, graphically shown and commented. The influence of the higher catalyst layer porosity in the wall area upon the temperature distribution in the reactor is studied. The existence of two different regimes is shown - a high temperature one in the middle part of the layer and a low temperature one in the high porosity area of the layer in contact with the reactor wall. This leads to not very effective usage of the catalyst in these parts of the catalyst layer in the reactor. This simulation can be used for better understanding and controlling of the examined catalytic process.

KEY WORDS: Catalytic reactor, transient conditions, mathematical modelling, temperature distribution.
\end{abstract}

\section{Introduction}

One of the possible methods for the realization of catalytic processes in transient conditions is the method suggested by Boreskov and Matros [1, 2, 3 ] of the catalytic change in the direction of the reaction mixture feed into the immobilized catalyst layer. This is the case when cold gas flow is fed into a layer of preheated catalyst, thus creating favourable condition for the formation of a heat front moving to the direction of gas flow filtration. The periodic change in the direction of the reaction mixture flow allows a restriction of the front's motion in the catalyst layer and a simultaneous formation of high-temperature zone inside it, which is large enough to allow the chemical process to occur. The 
direct heat exchange between the hot catalyst and the cold gas flow favours the heat transfer processes in the layer. This fact allows the usage of the transient method to process, where the quantity of the generated heat by the reaction is insufficient for the development of an auto thermal work regime of the catalyst reactor, according to the classical steady-state schemes.

There have been published theoretical and experimental elaborations, investigating the above mentioned transient method applied for model reactions (Matros [4], Boreskov [5]) or concrete catalytic processes such as $\mathrm{SO}_{2}$ oxidation (Boreskov [6, 7]; [2, 3], Matros [10], Sapundzhiev [11, 12, 13]), [27] neutralization of waste gases, containing organic admixture (Boreskov [9], Matros [14], Eigenberger [15], Sapundzhiev [16]), nitrogen oxides reduction (Bobkova [17]) and others. These publications reveal some of the advantages of the transient method to the traditional methods for the realization of such processes. At the same time, the specific peculiarities of the transient method (a periodic change in the direction of a cold reaction flow through a hot catalyst layer) lead to enhancement of the influence of some factors, which in the case of conventional processes are not essential for the optimal work regime of the catalytic reactors. Thus, in (Boreskov's work [9]) at $\mathrm{SO}_{2}$ catalytic oxidation essential radial temperature gradients are fixed near the wall side of the catalytic layer in the semi-industrial scale reactors, which according to the authors, is due to substantial heat losses through the reactor's walls. For the mathematical nodes, used in the above mentioned works, it is suggested that there are not any radial temperature and concentration gradients, because of which, these models cannot explain the radial non-homogeneities, observed by Boreskov.

The influence of the heat losses through the wall upon the work regimes of a catalytic reactor with a periodic change in the direction of the reaction mixture feed is investigated in a previous article (Sapundzhiev [12]), with the help of a two-phase heterogeneous model, accounting for the radial mass and heat transfer as well as the heat exchange at the reactor's walls. It is shown, that the heat losses at the reactor walls essentially influence the work regime of the short diameter reactions (less than $2 \mathrm{~m}$ ) and in case of adiabatic rise of temperature $\left(\Delta T_{a d}\right)$ up to $50^{\circ} \mathrm{C}$ as a result of a chemical reaction.

In Boreskov's work [7], the presence of radial temperature gradients even at higher $\Delta T_{a d}$ is mentioned. This shows that when describing the processes in the reactors with a periodic change in the direction of the reaction mixture feed, there are other factors except heat losses to be considered.

The aim of the present paper is to investigate the influence of the increased poreness of the catalyst layer near the walls upon the temperature distribution in a fixed catalyst layer reaction working in transient conditions 
through a periodic direction change of the reaction mixture feed.

\section{Statement of the problem}

A cylinder tube of radius $R$ and length $L$ is filled with catalyst in the form of equal hard porous spheres of radius $d_{c}$ (Fig. 1). The reacting mixture is fed through the cross-section $Z=0$ at moments $t=k \cdot t_{c}^{\prime}(k=0,1,2, \ldots)$ with velocity $V\left(R^{\prime}\right)$ and through the cross-section $Z=L$ at moments $t=$ $k \cdot t_{c}^{\prime}+t_{c}^{\prime} / 2(k=0,1,2, \ldots)$, also with velocity $V\left(R^{\prime}\right)$. Thus, an alternating reaction mixture feeding is realized at the different ends of the reactor, which gives a periodically changing picture of the mixture flow in the catalytic layer. The time for the accomplishment of one cycle $t_{c}^{\prime}$ is a constant, during the work of the reactor.

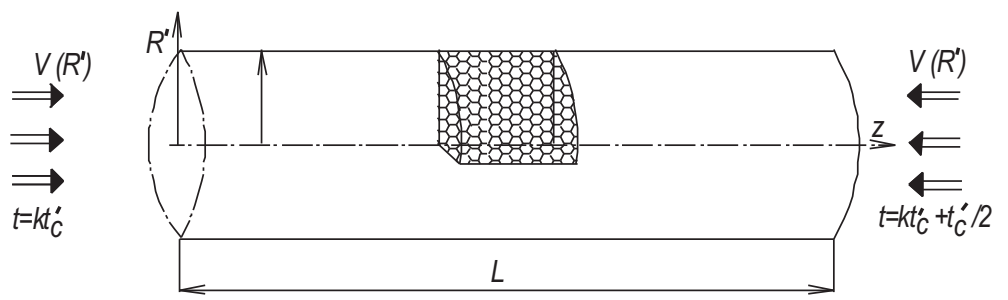

Fig. 1. Diagram of catalyst reactor

A two-dimensional pseudo-homogeneous model, described by Fromed [18] is used for the description of the temperature fields in the reactor. This model includes the following assumptions:

- Mass and heat transfer takes place in radial direction, through effective diffusion and heat exchange according to Fick's and Fourier's laws, respectively.

- Because of the high gas mixture velocity, which is typical for industrial reactors, the effective axial heat exchange and mass transfer in axial direction are neglected and thus, in axial direction, only convective mass and heat transfer are taken into account.

- The catalyst grains are isothermal and the processes proceeding on their internal surface are quasi-steady. This assumption is correct for industrial processes proceeding at almost atmospheric pressure.

- The reaction is exothermal and as a result a certain heat quantity is generated.

The equations describing the process (in a cylinder co-ordinate system 
$\left(R^{\prime}, \varphi, Z\right)$ under these assumptions (see Fig. 1) are

(1) $\varepsilon\left(R^{\prime}\right) \frac{\partial C}{\partial t}+V\left(R^{\prime}\right) \frac{\partial C}{\partial Z}=\frac{1}{R^{\prime}} \frac{\partial}{\partial R^{\prime}}\left(D_{r} R^{\prime} \frac{\partial C}{\partial R^{\prime}}\right)-k_{0} C \exp \left(-E / R_{g} T\right)$

$$
\begin{aligned}
& {\left[\left(1-\varepsilon\left(R^{\prime}\right)\right) \rho_{k} c_{p}+\varepsilon\left(R^{\prime}\right) \rho_{g} c_{g}\right] \frac{\partial T}{\partial t}+V\left(R^{\prime}\right) \frac{\partial T}{\partial Z}=} \\
& =\frac{1}{R^{\prime}} \frac{\partial}{\partial R^{\prime}}\left(\Lambda_{r} R^{\prime} \frac{\partial T}{\partial R^{\prime}}\right)+Q k_{0} C \exp \left(-E / R_{g} T\right) .
\end{aligned}
$$

The poreness of the layer $\varepsilon$ is supposed to depend on $R^{\prime}$ only and is equal in all cross-sections of the reactor $Z=Z_{*}, 0 \leq Z_{*} \leq L$.

The boundary condition, at which a solution of this system is looked are:

$$
\begin{gathered}
t=0: T=T_{k}, \quad V=V\left(R^{\prime}\right), \quad C=0 ; \\
t=k \cdot t_{c}^{\prime}(k=0,1,2, \ldots) \text { and } Z=0: V=V\left(R^{\prime}\right), C=C_{0}, T=T_{0} ; \\
t=k \cdot t_{c}^{\prime}+t_{c}^{\prime} / 2(k=0,1,2, \ldots) \text { and } Z=L: \\
\frac{\partial C}{\partial R^{\prime}}=\frac{\partial T}{\partial R^{\prime}}=0 \text { at } R^{\prime}=0 ; \\
\frac{\partial C}{\partial R^{\prime}}=0, \quad-\Lambda_{r} \frac{\partial T}{\partial R^{\prime}}=A\left(T-T_{*}\right) \text { at } R^{\prime}=R .
\end{gathered}
$$

The last condition is the heat balance upon the isolated wall of the reactor.

The dimensional coefficients of heat condition through the isolated wall $A$ for thermally isolated asbest cotton reactors wall is:

$$
A=0.110^{-5} \mathrm{kkal} / \mathrm{m}^{2} . s .{ }^{\circ} \mathrm{C} .
$$

In order to transform the system (1) with the boundary and initial conditions (2) into dimensionless form, we use the following: $c=C / C_{0}, B=$ $T / T_{0}, B_{k}=T_{k} / T_{0}-1, \theta_{*}=T_{*} / T_{0}-1, z=Z / z_{0}, z_{l}=L / z_{0}, z_{*}=Z_{*} / z_{0}$, $v(r)=V\left(R^{\prime}\right) / V_{0}, t=V_{0} / z_{0}, t=\tau \cdot V_{0} / z_{0}, c_{p}=\frac{\rho_{0} c_{0}}{\rho_{g} c_{g}}, q=Q C_{0} /\left(\rho_{g} c_{g} T_{0}\right)$, $\left.\left.\alpha_{r}=E / R_{g} T_{0}\right),\right) k=z_{0} \cdot k_{0} \cdot \exp \left(-E / R_{g} T_{0}\right) / V_{0}, d_{r}=z_{0} D_{r}\left(R^{\prime}\right) / R^{2} V_{0}, \lambda_{r}=$ $z_{0} \Lambda_{r}\left(R^{\prime}\right) / R^{2} V_{0}, r=R^{\prime} / R, t=t_{c}^{\prime} z / V_{0}, \alpha=R A / \lambda_{r}(R)$.

The process taking place in the reactor is considered exothermal, the reaction being of the first order, whose velocity is $k_{0} C \exp (-E / T R)$. 
Using the mentioned above quantities system (1) and boundary and initial conditions (3), can be written in the form:

$$
\begin{gathered}
\frac{\varepsilon(r)}{c_{p}} \frac{\partial c}{\partial \tau}+v(r) \frac{\partial c}{\partial z}=\frac{1}{r} \frac{\partial}{\partial r}\left(d_{r} r \frac{\partial c}{\partial r}\right)-k \cdot c \cdot \exp \left(\alpha_{r} \frac{\theta}{1+\theta}\right) \\
{\left[1-\varepsilon(r)+\frac{\varepsilon(r)}{c_{p}}\right] \frac{\partial \theta}{\partial \tau}+v(r) \frac{\partial \theta}{\partial z}=\frac{1}{r} \frac{\partial}{\partial r}\left(\lambda_{r} r \frac{\partial \theta}{\partial r}\right)+q . k . C \cdot \exp \left(\alpha_{r} \frac{\theta}{1+\theta}\right)} \\
\tau=0: \theta=\theta_{k}, \quad v=v(r), \quad c=0, \quad \theta=0 \\
\tau=k . t_{c}(k=0,1,2, \ldots) \text { and } z=0: v=v(r), c=1, \theta=0 \\
\tau=k . t_{c}+t_{c} / 2(k=0,1,2, \ldots) \text { and } z=z_{l}: \\
\frac{\partial c}{\partial r}=\frac{\partial \theta}{\partial r}=0 \text { at } r=0 ; \frac{\partial c}{\partial r}=0,-\frac{\partial \theta}{\partial r}=\alpha\left(\theta-\theta_{k}\right) \text { at } r=1 .
\end{gathered}
$$

It goes out that $c_{p} \approx 10^{3}$, if the radial quantities of the parameters are substituted in the expression for $c_{p}$. A conclusion could be made from the first equation of (3), that the time of the concentration wave movement, which is of the following value: $L \varepsilon\left(R^{\prime}\right) / V_{0} \sim 2$ seconds is essentially smaller than the time necessary for the heat wave movement $L(1-\varepsilon) / V_{0} \sim 1.5 \mathrm{~h}$. It is because the turning occurs in $0.5 \sim 1$ hour, in equations (3) all its parts, which are of the same order as $c_{p}^{-1}$ could be neglected. Then, these equations turn into:

$$
\begin{gathered}
v(r) \frac{\partial c}{\partial z}=\frac{1}{r} \frac{\partial}{\partial r}\left(d_{r} r \frac{\partial c}{\partial r}\right)-k . c \cdot \exp \left(\alpha_{r} \frac{\theta}{1+\theta}\right) \\
{\left[(1-\varepsilon(r)] \frac{\partial \theta}{\partial \tau}+v(r) \frac{\partial \theta}{\partial z}=\frac{1}{r} \frac{\partial}{\partial r}\left(\lambda_{r} r \frac{\partial \theta}{\partial r}\right)+q . k . C \cdot \exp \left(\alpha_{r} \frac{\theta}{1+\theta}\right) .\right.}
\end{gathered}
$$

Different methods have been used for the modelling of the poreness of the layer near to the wall of the apparatus. Vortmayer [19] and Vafai [20] suggest that the porosity of the layer beside the wall is exponentially changing with the radius. In order to consider the non-uniform poreness in an adsorbing apparatus, Naumov et al. [21] a two zone model is used, which suppose a steplike profile of the poreness. The poreness beside the wall is higher than the one in the central part, the heat and mass exchange between the two zones is neglected.

This paper is dedicated to study the influence of boundary effects near the reactor walls, which are due to the different poreness of the layer in radial 
direction. Two model dependency relations $\varepsilon(r)$ and $v(r)$ have been used: in the first case $\varepsilon(r)=\varepsilon_{0}$ and $v(r)=1$ and in second case the change in the porosity in the domain, besides the wall, is taken into account, similar to that shown by Vortmayer [19].

$$
\begin{gathered}
\varepsilon(r)=\varepsilon_{0}\left[1+\exp \left(1-2 \frac{R(1-R)}{\xi d_{c}}\right)\right], \\
v(r)=g(r) / 2 \int_{0}^{1} r g(r) d r \\
g(r)=1-\exp \left(4 n /(4-n) \frac{R(1-R)}{d_{c}}\right)\left(1-n \frac{R(1-R)}{d_{c}}\right) .
\end{gathered}
$$

For $0.1 \leq R e_{p} \leq 1$ :

$$
n=112.5-26.31 R e_{p}-10.97 R e_{p}^{2}-0.1804 R e_{p}^{4}
$$

For $1 \leq R e_{p} \leq 1000$ :

$$
n=-1803+201.61\left(\ln R e_{p}+4\right)-3737\left(\ln R e_{p}+4\right)^{1 / 2} .8399\left(\ln R e_{p}+4\right)^{1 / 3},
$$

and $1000 \leq R e_{p}: n=27$.

Consider a model reaction of catalytic oxidation of a buten mixture, the catalyst being $\mathrm{Cu}-\mathrm{Cr}$ of type IKT-12-8. The kinetic characteristics are derived under assumption, that the reaction is of first order and because of the excess of the oxide the reaction velocity does not depend on its concentration (Popovskii et al. [22]). The influence of the inner diffusive resistance is taken into account by using the observed kinetic parameters for the corresponding dimensions of the catalyst grains, using methods suggested by Malinovskaya and others, [23]. The frequency factor and the activation energy of this reaction are $k_{0}=0.32 .10^{6} \mathrm{sec}^{-1}, E=12.82 \mathrm{kkal} / \mathrm{mol}=53.71 \mathrm{~kJ} / \mathrm{mol}$, for spherical catalyst grains of diameter $d_{c}=5 \mathrm{~mm}$.

For calculating the effective coefficient of radial diffusion $D_{r}$, the following criteria relation (for spherical catalyst grains and for $D / d_{c}>15$ is used (Aerov et al. [24]):

$$
\begin{gathered}
\frac{D_{r}}{D_{m}}=0.28+0.09 \cdot R e_{e} \cdot S c \\
R e_{e}=\frac{v_{e} \cdot d_{c}}{\nu}=\frac{4 . v_{c}}{a \cdot \nu}, v_{e}=v(r) / e(r), a=\frac{6}{d_{c}}[1-\varepsilon(r)]\left[\mathrm{m}^{2} / \mathrm{m}^{3}\right], S c=\frac{\nu}{D_{m}} .
\end{gathered}
$$


For calculating the effective coefficient of radial heat conducting $\lambda_{r}$, we use the criteria relation (Kukharni [25], De Wash and Froment[23]): $\lambda_{r}=$ $\lambda_{s t}+\lambda_{\text {din }}$, where

$$
\begin{gathered}
\frac{\lambda_{s t}}{\lambda_{g}}=\varepsilon+(1-\varepsilon) \frac{1}{0.12+\frac{2}{3} \frac{\lambda_{g}}{\lambda_{c}}} \\
\lambda_{\text {din }}=\frac{0.0025}{1+46\left(2 R / d_{c}\right)^{2}} R e^{\prime}, \quad R e^{\prime}=d_{c} \cdot v / \nu .
\end{gathered}
$$

The system (6) with the boundary condition (4) is solved numerically by a finite differences method with iterations (Samarskii [26]).

\section{Results from modelling}

Two series of numerical experiments were provided, to define the influence of the side effects. In the firs one, it was accepted that the porosity $\varepsilon(r)$ and the local velocity of the flow $v(r)$ are constant at the apparatus's radius. The second case, they were counted according to equation (6). The width of the wall side area had been changed in the interval $(5-20) d_{c}$, at which it varied from 25 to $100 \mathrm{~mm}$. The calculations were made at a constant length of the catalyst layer $L=2 \mathrm{~m}$, the reactor diameter $D=2 \mathrm{~m}$, the duration of the cycle of turning on the reaction feed $t_{c} / 2=27 \mathrm{~min}$ and the diameter of the catalyst grain $d_{c}=5 \mathrm{~mm}$. During this process, the entrance reaction mixture concentration was varied in the interval $\left(0.125-0.625 \mathrm{~mol} / \mathrm{m}^{3}\right)$, which corresponds to the adiabatic rise of the temperature, as a result of a chemical reaction in the interval $\left(20^{\circ} \mathrm{C}-100^{\circ} \mathrm{C}\right)$.

The results from this modelling are shown in Figs $2-6$. In all figures, the temperature profiles are built at moments $\tau=t_{c} / 4$ (in the middle of the semi cycle of the already established regime).

Figure 2 shows the maximum temperature dependency in time: curve 1 shows the change in maximum temperature in the reactor, at the condition that the porosity $\varepsilon(r)$ and the local velocity of the flow $v(r)$ are varied at the cross-section of the apparatus (model 1 ) and curve 2 is at $\varepsilon(r)=$ const and $v(r)=$ const (model 2).

It becomes obvious from the figure, that in 5 or 6 cycles in the reactor a periodically changing temperature regime is established. It is essential to point out, that the maximum temperature in model 1 is about $10^{\circ} \mathrm{C}$ higher than that in the model 2. At first sight, it is quite unreasonable. To explain the fact, Fig. $3 a$ shows the temperature fields in the reactor volume according to model 1 and Fig. $3 b$ - to model 2. It is obvious, that the structure of the temperature 


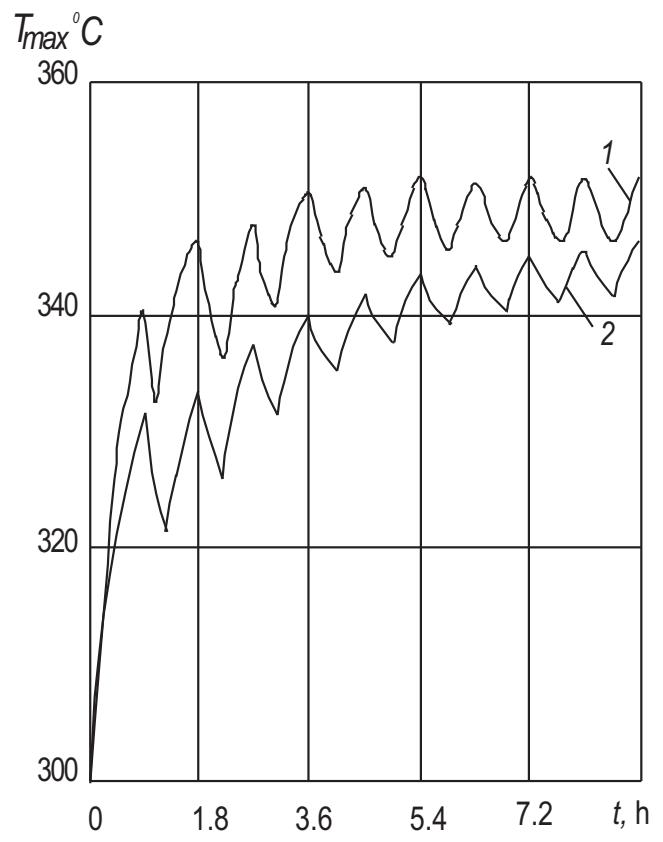

Fig. 2. Maximum temperature dependence on time at $C_{0}=0.125 \mathrm{~mol} / \mathrm{m}^{3}$, $T_{0}=20^{\circ} \mathrm{C}, t_{c / 2}=0.9 \mathrm{~h}$, the width of the wall area $5 . d_{c}$. a) according to model $1[\varepsilon(r) \neq$ const and $v(r) \neq$ const]; b) according to model $2[\varepsilon(r)=$ const and $v(r)=$ const]

distribution in the reactor is absolutely different in the two models. According to model 1 , because of the higher gas velocity in the wall areas of the catalyst layer and heat losses through the reactor wall in these parts of the layer, a consideration drop in temperature is observed.

At dimensionless radius $r=0.02$ from the apparatus wall, an area with a lower than $200^{\circ} \mathrm{C}$ temperature is formed, through which the gas passes without being chemically engaged.

In the central part of the layer, the high temperature zone is preserved in which the rate of conversion is practically $100 \%$. In this way, there are two different regimes in the reactor: a high temperature one in the middle part of the catalyst layer and a low temperature one in the high porosity area, near the reactor wall. Except that, model 1 demonstrates a temperature maximum along the apparatus radius.

It can be understood better from Fig. 4, where the radial temperature profile of the two models at the cross-section along the dimensionless length of the layer $z=0.5$ is shown.

The temperature maximum is at $r=0.14$, that is just in the front of high porosity area. This, according to us, is due to the fact that there is a supplementary feed of substance from the low temperature area on account of 

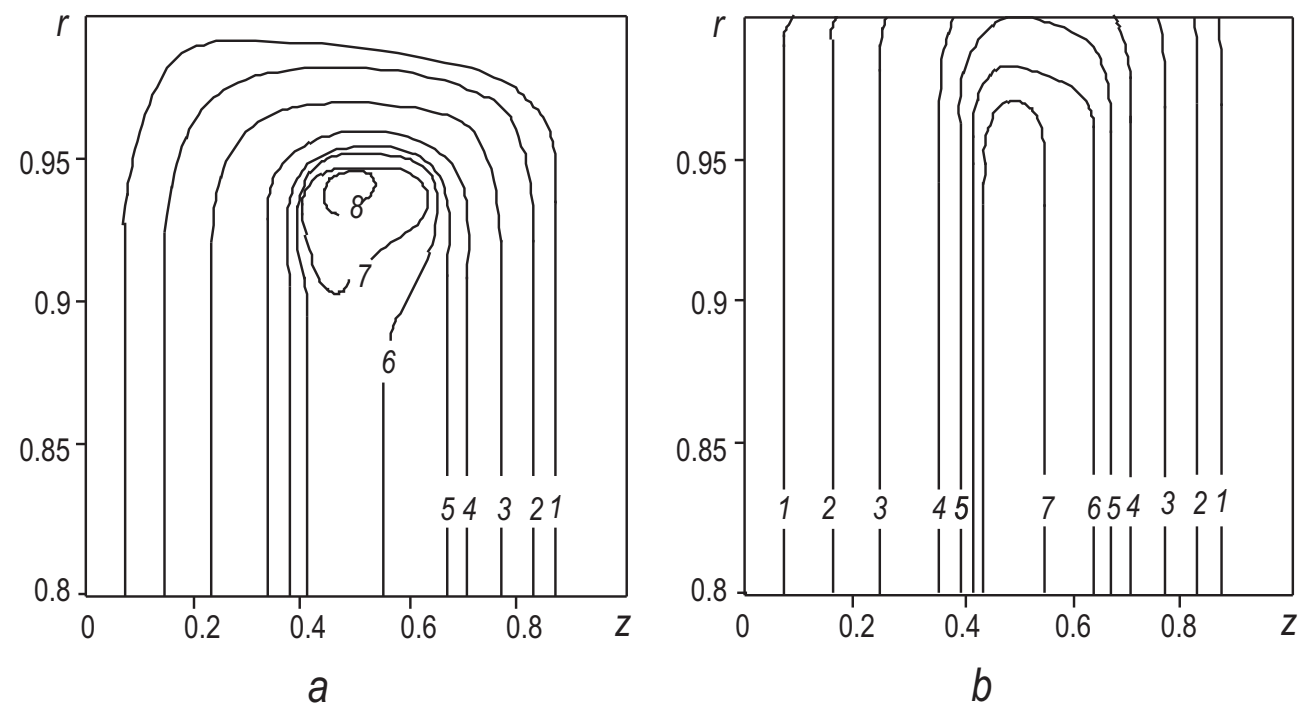

Fig. 3. Temperature distribution in reactor volume at $C_{0}=0.125 \mathrm{~mol} / \mathrm{m}^{3}, T_{0}=20^{\circ} \mathrm{C}$, $t_{c / 2}=0.9 \mathrm{~h}$, the width of the wall area $5 . d_{c}$, isotherms $1-50{ }^{\circ} \mathrm{C}, 2-100{ }^{\circ} \mathrm{C}, 3-200^{\circ} \mathrm{C}$, $4-310^{\circ} \mathrm{C}, 5-330^{\circ} \mathrm{C}, 6-337^{\circ} \mathrm{C}, 7-340^{\circ} \mathrm{C}, 8-345^{\circ} \mathrm{C}$. a) according to model 1 $[\varepsilon(r) \neq$ const and $v(r) \neq$ const $] \mathrm{b})$ according to model $2[\varepsilon(r)=$ const and $v(r)=$ const $]$

the radial gas diffusion (the gas diffusion in radial direction). When this gas reaches the high temperature area, it reacts chemically as a result of which heat is generated, which is the reason for the above mentioned maximum. When comparing curves 1 and 2 (Fig. 4), the relative share of the heat losses through the reactor walls can be defined. The drop in temperature near the wall according to model 2 - curve 2 is due to heat losses through the reactor wall. The expending of the high porosity area to $10 d_{c}$ to $20 d_{c}$ shows the same results as in the case at which the width of the near-the-wall area is $5 d_{c}$ and at the sane time the effects described above are more definitely expressed. This is proved by Fig. 5, where the temperature profiles along the length of the reactor in the central part of the layer are shown $(r=0)$. Curve 1 is presented at width of the wall area of $20 d_{c}$ and curved $2-$ at $5 d_{c}$. The further widening of the high porosity zone leads to decreasing the maximum temperature in the central area but at the same time the high temperature area is getting wider.

The influence of the initial concentration of organic substance in the gas mixture is shown in Fig. 6, which also demonstrates the temperature fields at initial concentration $C_{0}=0.187 \mathrm{~mol} / \mathrm{m}^{3}$ to model $1-$ Fig. $6 a$ and to model 2 - Fig. $6 b$. These figures demonstrate that the growing of the 

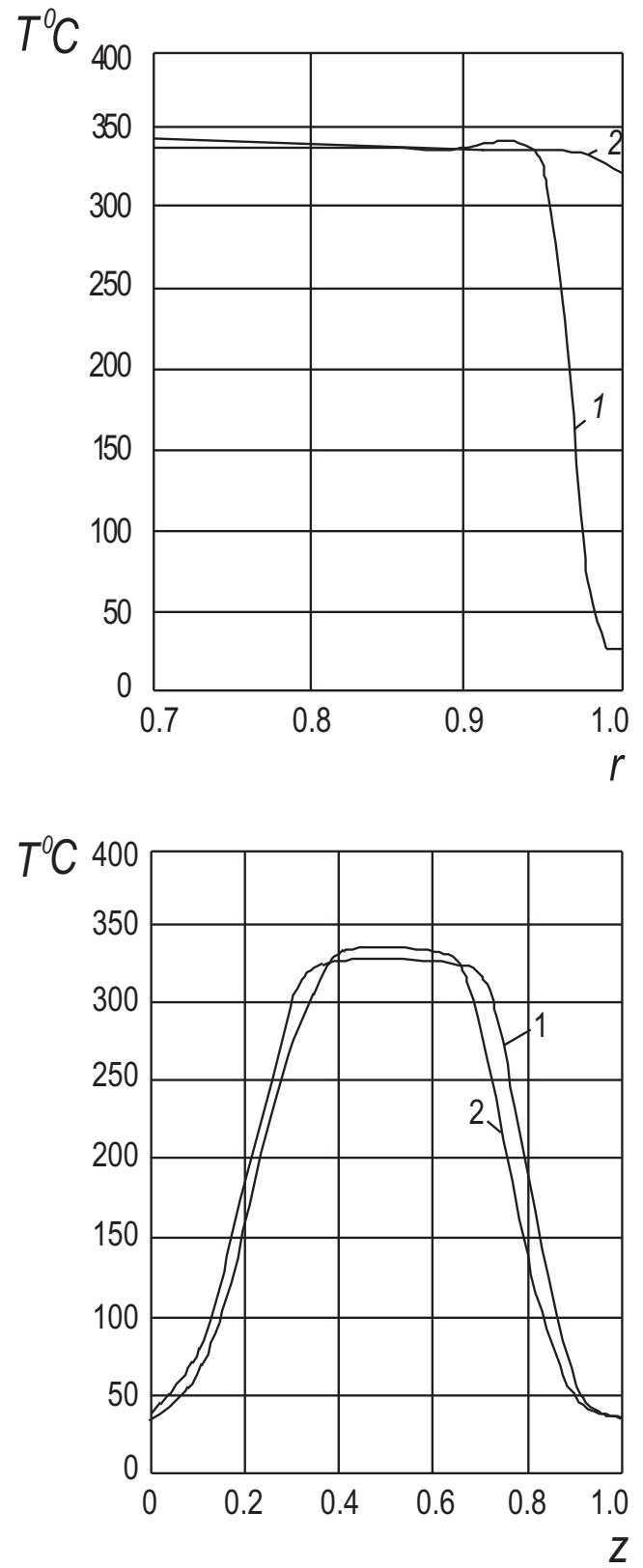

Fig. 4. Radial temperature profiles at $z=0.5, C_{0}=0.125 \mathrm{~mol} / \mathrm{m}^{3}, T_{0}=$ $20{ }^{\circ} \mathrm{C}, t_{c / 2}=0.9 \mathrm{~h}$, the width of the wall area $5 . d_{c}$ a) according to model 1 $[\varepsilon(r) \neq$ const and $v(r) \neq$ const $] \mathrm{b})$ according to model $2[\varepsilon(r)=$ const and $v(r)=$ const]
Fig. 5. Temperature profiles along the length of the reactor in the central part of the layer $(r=0), C_{0}=0.125$ $\mathrm{mol} / \mathrm{m}^{3}, T_{0}=20{ }^{\circ} \mathrm{C}, t_{c / 2}=0.9 \mathrm{~h}$, the width of the wall area $20 . d_{c}-$ curve 1 and $5 . d_{c}-$ curve 2 


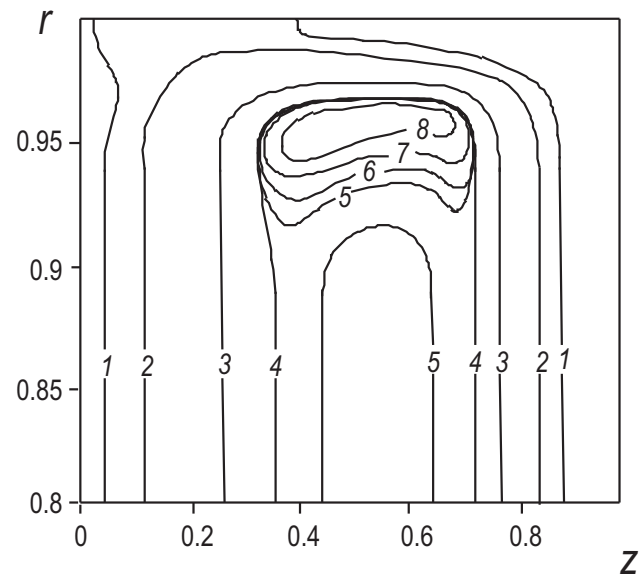

a

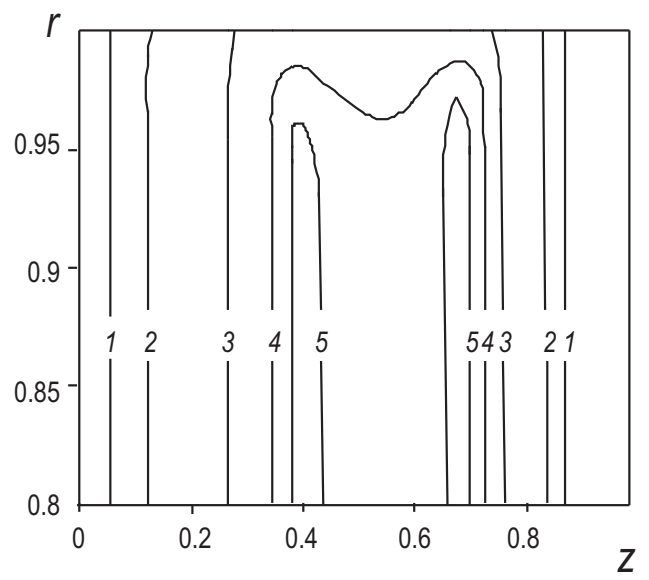

$b$

Fig. 6. Temperature distribution in reactor volume according to model 1 (a) and according to model 2 (b), at $C_{0}=0.187$; isotherms $1-50{ }^{\circ} \mathrm{C}, 2-100{ }^{\circ} \mathrm{C}, 3-300{ }^{\circ} \mathrm{C}$, $4-360{ }^{\circ} \mathrm{C}, 5-365{ }^{\circ} \mathrm{C}, 6-367{ }^{\circ} \mathrm{C}, 7-370{ }^{\circ} \mathrm{C}, 8-375{ }^{\circ} \mathrm{C}$

initial concentration of organic substrate leads to higher maximal temperature in the reactor, compared to the maximum temperature at $C_{0}=0.125 \mathrm{~mol} / \mathrm{m}^{3}$ (Fig. 3a,b). Another essential difference is the presence of two maximums at the layer length, which are observed either in model 1 and model 2. To our understanding, this is due to heat losses through the reactor wall.

The presence of higher organic content in the gas mixture creates conditions for widening of the high temperature area, which leads to heating of the border part of the catalyst layer in the gas mixture feed direction. The high temperatures area begins to move in the opposite direction, when changing the direction of the gas mixture feed. The rich gas mixture at the beginning of the layer "meets" the heated catalyst layer from the last cycle and in comparatively narrow area of the catalyst it is heated to the reaction temperature. As a result of the chemical reaction, a definite quantity of heat is realized. In the rest of the layer practically no chemical reaction occurs, but the heat is predominantly being exchanged through the reactor walls into the environment. As a result, a temperature drop in the central part is observed.

\section{Conclusions}

The modelling shows, that the temperature distribution in a reactor with an immobilized catalyst layer, working in transient conditions through a 
periodic change into the reaction mixture feed direction according for the nearthe-wall effects generated by the higher porosity and the local gas velocity along the cross-section of the apparatus is essentially different from that at $\varepsilon(r)=$ const and $v(r)=$ const. The essential differences in the temperature of the cold gas flow and the heated catalyst layer and the higher velocity of the gas mixture in these areas create favourable conditions for generation a low temperature area in which the temperature is lower than the reaction temperature and in this area there is no chemical reaction in process. This results in poor usage of the catalyst in such areas.

In conclusion, we will point out, that considering the radial non-homogeneities within the reactors of immobilized catalyst layer, working in transient conditions through a periodic change in the reaction mixture feed direction, in contrast to the classical (conventional) stationary apparatuses, is of great importance for the exact prediction of the processes, according to these reactors and for their effective projecting and manipulating (controlling).

Acknowledgements. My special thanks to my colleagues and friends

Dr H. Sapundzhiev and Dr V. Kurdyumov for the useful discussions.

\section{REFERENCES}

[1] Boreskov, G. K., Yu. Sh. Matros, O. V. Kiselov, G. Bunimovich. A Implementation of Heterogeneous Catalytic Process in the Transient Regime. Docl. AN SSSR, 237 (1977), No. 1, 160-163.

[2] Boreskov, G. K., Yu. Sh. Matros. Fixed Catalyst Bed Reactors operated in Steady- and Unsteady- state Conditions. Recent Advances in the English Analytical of Chemistry Reaction Systems, (1984), 142-155.

[3] Boreskov, G. K., Yu. Sh. Matros, V. I. Lugavskoi, G. A. Bunimovich, V. I. Puzhilova. Transient Process of Deep Oxidation in a Fixed Bed Reactor. Teor. Osnovi Chim. Tekhnologii, 18 (1984), No. 3, 328-333 (in Russian).

[4] Matros, Yu. Sh. Cathalytic Processes in Non-steady Conditions. Zh. Vses. Khim. Ob. im Mendeleeva (USSR), 22 (1977), No. 5, 576-580 (in Russian).

[5] Boreskov, G. K., Yu. Sh. Matros, O. V. Kiselov. Implementation of Catalytic Processes in Unsteady Conditions: 1. Heat the Front in a Fixed Bed Catalyst. Kin. \& Katal., 20 (1979), 773-780 (in Russian).

[6] Boreskov, G. K., Yu. Sh. Matros. Flow Reversal of Reaction Mixture in a Fixed Catalyst Bed - a Way to increase the Efficiency of Chemical Processes. Applied Catalysis, 5 (1983), 337-343.

[7] Boreskov, G. K., Yu. Sh. Matros. Unsteady-state Performance of Heterogeneous Catalytic Reactions. Catal. Rev. Sci. Eng., 25 (1983), No. 4, 551-590. 
[8] Boreskov, G. K., G. A. Bunimovich, Yu. Sh. Matros, A. A. Ivanov. Implementation of Catalyst Processes in Unsteady Conditions. Kinetics and Catalysis, 23 (1982), No. 2, 402-406.

[9] Boreskov, G. K., Yu. Sh. Matros, G. A. Bunimovich et al. Nonstady state Processes in Chemical Reactors, Novosibirsk, 1982, 80-84.

[10] Matros, Yu. Sh., A. S. Noskov, V. A. Chumachenko, O. V. Goldman. Theory and Application of Unsteady Catalytic Detoxication of Effluent Gases from Sulfur Dioxide, Nitrogen Oxides and Organic Compounds. Chemical Engineering Science, 43 (1988), No. 8, 2061-2066 .

[11] Sapundzhiev, Chr., G. A. Bunimovich, Yu. Sh. Matros. Mathematical Modelling of Non-stationary Method for Sulfuric Acid Production from Low-concentrated Metallurgy Gases. Commun. Dept. Chem. Bulg. Acad. Sci., 17 (1984), 298-304.

[12] Sapundzhiev, Chr. Unseady-State Method for Sulphuric Acid Production Low Concentrated $\mathrm{SO}_{2}$ Gases, Ph. D. Thesis, USSR, Novosibirsk, 1988.

[13] Sapundzhiev, Chr., G. Grozev, D. Elenkov. Influence of Geometric and Thermophysical Properties of Reaction Layer on Sulphur Dioxide Oxidation in Transient Conditions. Chem Eng. \&3 Technol, 13 (1990), No. 2, 131-135.

[14] Matros, Yu. Sh., A. S. Noskov, U. A. Chumachenko, O. V. Goldman. Theory and Application of Unsteady Catalytic Detoxication of Effluent Gases From Sulfur Dioxide. Chem. Eng. Sci., 43 (1988), No. 8, 2061-2066.

[15] Eigenberger, G., U. Nieken. Catalytic Combustion with Periodic Flow Reversal. Chem. Eng. Sci., 43 (1988), No. 8, 2109-2115.

[16] Sapundzhiev, Chr., G. Grozev, D. Elenkov. Non-steady-state Catalytic Decontamination of Waste Gases. Chem Eng. 83 Technol., 14 (1991), No. 3, 209-212.

[17] Bobrova, L. N., E. M. Slavinskaya, A. S. Noskov, Yu. Sh. Matros. Unsteady-state Performance of $\mathrm{NO}_{\mathrm{x}}$ Catalytic Reduction by $\mathrm{NH}_{3}$. Reaction Kinetics and Catalisis letters, 37 (1988), No. 2, 267-272.

[18] Froment, G. F. Heat and Mass Transfer in Fixed Bed Catalyting Reactors, 5 International summer school "Modelling of Heat and Mass Transfer Processes and Chemical Reactor", Bulgaria, 1985, 65-72.

[19] Vortmeyer, D. Schuster Evaluation of Steady Flow Profiles in Rectangular and Circular Packed Beds by a Variational Method. J. Chem. Eng. Sci., 38 (1983), No. 10, 1691-1699.

[20] Vafai, K., B. L. Alkire, C. L. Tien. An Experimental Investigation of Heat Transfer in Variable Porosity Media. Trans ASME Journal Heat Trans., 107 (1985), No. 3, 642-647.

[21] Malinovskaya, O. A., V. C. Beskov, M. G. Slinko. Models of Catalytic Processes on Porous Grains, Novosibirsk, 1975, p. 264.

[22] Aerov, M. E., O. M. Todev, D. A. Narinskir. Apparatuses with Stady state Grain Layer, Leningrad, 1979, p. 173.

[23] Kulkami, B. D., L. K. Doraiswami. Estimation of Effective Transport Properties in Packed Bed Reactors. Catal. Rev. Sci. Eng., 22 (1988), No. 3, 431-483. 
[24] Samarskit, A. A. Theory of Discrete Differential Equations, Moskow, Nauka, 1978.

[25] De Wash, A. P., G. F. Froment. Heat Transfer in Packed Bed. Chem. Eng. Sci., 27 (1972), 567-576.

\section{Nomenclature}

$A$ - dimensional coefficient of heat conduction through the isolated wall

$a$ - specific surface of the layer $\left[\mathrm{m}^{2} / \mathrm{m}^{3}\right]$

$\mathrm{C}_{0}$ - initial concentration $\left[\mathrm{mol} / \mathrm{m}^{3}\right]$

$C$ - concentration, $\left[\mathrm{mol} / \mathrm{m}^{3}\right]$

$c$ - dimensionless concentration

$c_{c}$ - dimensionless catalyst concentration

$c_{g}$ - dimensionless gas concentration

$c_{p}$ - dimensionless specific special heat capacity of the medium

$D$ - diameter of the reactor, $D=2 R,[\mathrm{~m}]$;

$D_{r}$ - coefficient of effective radial diffusion

$D_{m}$ - coefficient of molecular diffusion of the reacting component in the air

$d_{r}$ - dimensionless effective coefficient of radial diffusion

$d_{c}$ - radius of the catalyst grains, $[\mathrm{mm}]$

$E$ - activation energy of the reaction, $[\mathrm{kJ} / \mathrm{mol}]$

$k$ - dimensionless frequency factor

$k_{0}$ - frequency factor, $\left[\mathrm{sec}^{-1}\right]$

$R$ - radius of the reactor, $[\mathrm{m}]$

$R^{\prime}$ - radial position, $[\mathrm{m}]$

$R_{g}$ - the gas constant

$R e_{p}$ - Reynolds number, $R e_{p}=v_{0} \cdot d_{c} / \nu$

$R e^{\prime}$ - Reynolds number, $R e^{\prime}=v \cdot d_{c} / \nu$

$L$ - length of the reactor, $[\mathrm{m}]$ 
Temperature Non-Homogeneieties in a Catalytic Reactor ...

$Q$ - the heat effect of the reaction

$q$ - dimensionless thermal effect of the reaction;

$S c$ - Schmidt number, $S c=D_{m} / \nu$

$T_{k}$ - initial temperature of the catalyst, ${ }^{0} \mathrm{C}$

$t_{c}{ }^{\prime}$ - time for the accomplishment of one cycle, [h]

$t_{c}$ - dimensionless time for the accomplishment of one cycle,

$V$ - velocity of reaction mixture, $[\mathrm{m} / \mathrm{s}]$

$v$ - dimensionless velocity

$V_{0}$ - average velocity, $V_{0}=\frac{2}{R^{2}} \int_{0}^{R} r . V(r) d r,[\mathrm{~m} / \mathrm{s}]$

$v_{e}$ - velocity of the mixture between the catalyst grains, $[\mathrm{m} / \mathrm{s}]$

$Z$ - axial position, $[\mathrm{m}]$

$z$-dimensionless axial position,

$z_{0}$ - characteristic meaning of the length of the reactor.

\section{Greek letters}

$\varepsilon$ - layer porosity

$\varepsilon_{0}$ - initial layer porosity

$\rho_{c}$ - density of the catalyst, $\left[\mathrm{kg} / \mathrm{m}^{3}\right]$

$\rho_{g}$ - density of the gas, $\left[\mathrm{kg} / \mathrm{m}^{3}\right]$

$\nu$ - kinematics viscosity coefficient

$\phi$ - angular coordinate

$\Lambda$ - coefficient of the effective radial thermal conductivity

$\lambda_{r}$ - dimensionless coefficient of effective radial thermal conductivity

$\lambda_{c}$ - heat conductivity coefficient for the catalyst

$\lambda_{g}-$ heat conductivity coefficient for the gas 
$\lambda_{\text {din }}$ - dynamic part of $\lambda_{r}$ according to Kuharny, 1980

$\lambda_{s t}$ - steady part of $\lambda_{r}$ according to Kuharny, 1980

$\xi$ - coefficient, equal to $5,10,20$

$\theta$ - dimensionless temperature

$\theta_{0}-$ dimensionless initial temperature

$\theta_{*}$ - dimensionless temperature of the outside

$\tau$-dimensionless time 